\title{
Molecular motor traffic: From biological nanomachines to macroscopic transport
}

\author{
Reinhard Lipowsky*, Yan Chai, Stefan Klumpp, Steffen Liepelt, Melanie J.I. Müller \\ Max-Planck-Institute of Colloids and Interfaces, Science Park Golm, 14424 Potsdam, Germany
}

Available online 6 June 2006

\begin{abstract}
All cells of animals and plants contain complex transport systems based on molecular motors which walk along cytoskeletal filaments. These motors are rather small and have a size of $20-100 \mathrm{~nm}$ but are able to pull vesicles, organelles and other types of cargo over large distances, from micrometers up to meters. There are several families of motors: kinesins, dyneins, and myosins. Most of these motors have two heads which are used as legs and perform discrete steps along the filaments. Several aspects of the motor behavior will be discussed: motor cycles of two-headed motors; walks of single motors or cargo particles which consist of directed movements interrupted by random, diffusive motion; cargo transport through tube-like compartments; active diffusion of cargo particles in slab-like compartments; cooperative transport of cargo by several motors which may be uni- or bi-directional; and systems with many interacting motors that exhibit traffic jams, self-organized density and flux patterns, and traffic phase transitions far from equilibrium. It is necessary to understand these traffic phenomena in a quantitative manner in order to construct and optimize biomimetic transport systems based on motors and filaments with many possible applications in bioengineering, pharmacology, and medicine.
\end{abstract}

(C) 2006 Elsevier B.V. All rights reserved.

\section{Introduction}

Each cell of our body contains a huge number of small vesicles which exhibit complex patterns of intracellular traffic: some vesicles travel from the cell center to the periphery and vice versa, some shuttle between different organelles or cellular compartments. An extreme case is provided by the long-ranged transport of vesicles and organelles along the axons of our nerve cells as illustrated in Fig. 1. All of this traffic is based on two molecular components: cytoskeletal filaments, which form a complex network of rails, and molecular motors, which move along these filaments and act as nano-tractors.

In our cells as well as in those of all animals and plants, three types of proteins are used for cargo transport: kinesins, dyneins and myosins [1,2]. These motors have usually two heads, which they use as legs and by which they can bind to the filaments. In their bound states, these molecular motors undergo a cyclic sequence of conformational transitions, a so-called motor cycle, that enables them to transform the chemical energy of

\footnotetext{
${ }^{*}$ Corresponding author.

E-mail address: lipowsky@mpikg.mpg.de (R. Lipowsky).

URL: http://www.mpikg.mpg.de/th/.
} 


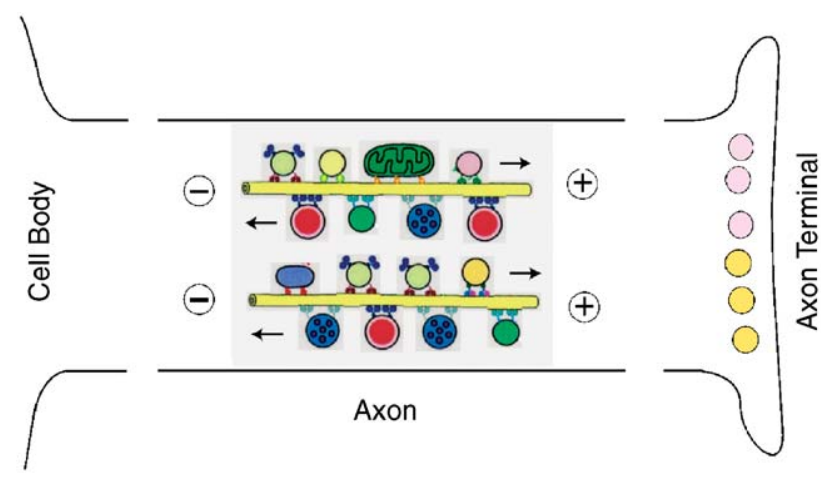

Fig. 1. Two-way traffic of molecular motors in axons which contains many microtubules, oriented with their plus ends towards the axon terminal and with their minus ends towards the cell body. The motors (indicated by small 'feet') are responsible for the transport of various types of cargo such as vesicles and organelles. Kinesin motors walk towards the plus end of the filaments, dynein motors towards the minus ends. Similar motor traffic is found in the cells of all animals and plants.

single adenosine triphosphate (ATP) molecules into discrete steps along the filament [3]. Two-headed motors walk in a 'hand-over-hand' fashion, i.e., by alternating steps in which one head moves forward while the other one remains bound to the filament [4-6]. Each step corresponds to a motor displacement of about $10 \mathrm{~nm}$, comparable to the size of the motor heads. If there is no shortage of ATP, the motor makes about 100 steps in $1 \mathrm{~s}$ which leads to a velocity of about $1 \mu \mathrm{m} / \mathrm{s}$. The absolute value of this velocity is not very impressive, but relative to its size, the motor molecule moves very fast: on the macroscopic scale, its movement would correspond to an athlete who runs $200 \mathrm{~m}$ in $1 \mathrm{~s}$ ! This is even more surprising if one realizes that the motor moves in a very viscous and noisy environment since it steadily undergoes thermally excited collisions with a large number of water molecules.

Indeed, because of this thermal noise, a single motor molecule falls off from the filament after it has made about a 100 steps. It has then covered a walking distance or run length of about $1 \mu \mathrm{m}$ [7] which is rather small compared to the long distances - centimeters or even meters - over which cargo is transported in cells and axons. One rather effective way to increase the run length is via cooperative transport of cargo particles by several motor molecules [8].

On length scales that are large compared to their run length, molecular motors with or without cargo undergo peculiar walks that consist of directed movements along the filaments interrupted by random, diffusive motion in the surrounding solution. These motor walks can be used for long-ranged cargo transport in tube-like compartments [9-11] and for active and enhanced diffusion of cargo particles in slab-like compartments [12]. It seems rather promising to use such motors in order to construct biomimetic transport systems, see, e.g., Refs. [13,14]. Possible applications are sorting devices for biomolecules and biocolloids, drug delivery systems that utilize motor transport within human cells, and motile components for nanoscale manufacturing.

In order to make many steps, the molecular motors must be strongly bound to the filament and the dynamic equilibrium between binding and unbinding must be shifted towards the bound state. The corresponding dissociation constant is of the order of a $100 \mathrm{nM}$ which corresponds to about 60 motors in a micrometer-sized volume of water. At these rather low concentrations, the motors already occupy a substantial fraction of the filaments' binding sites. As one further increases the motor concentrations, the filaments start to become overcrowded and the motor flux becomes reduced by traffic jams [9-11].

In vivo, the motor concentration can be varied by changing the expression level of the corresponding gene; using this method, jam-like density profiles have recently been induced and observed for fungal kinesin-like motors $[15,16]$. The effect of crowding is enhanced if the motors pull large cargoes such as membrane-bound organelles. Jam-like behavior of organelles has been observed in axons (Saxton, private communication); extreme cases induced by mutations of motors are accompanied by strong swelling of the axon [17,18]. It has also been proposed that such traffic jams are involved in a variety of neurodegenerative diseases [17,19]. Since traffic jams do not have an obvious biological function, one would expect that cells typically operate at motor 
concentrations which avoid overcrowded filaments. In addition, recent experiments with conventional kinesins show that it is even difficult to intentionally induce jams of these motors in vitro [20]. These experiments will be addressed further below in Section 6 .

Jamming or crowding arises from the mutual exclusion of motors which compete for the same space close to the filament. These generic motor-motor interactions also lead to a variety of motor density and flux patterns and to traffic phase transitions at which these patterns change in a qualitative and often abrupt manner. Examples are provided (i) by motor transport through tube-like compartments as one changes the motor densities at the two ends of an open tube [10,21-24], (ii) by motor entry into a half-open tube as one varies the ATP concentration [25], and (iii) by two-way traffic of two motor species that move in opposite directions as one changes the motor composition in the surrounding solution [26]. In this way, the motility of molecular motors covers many length and time scales as we have previously emphasized [27].

This article addresses these different motility regimes in a bottom-up manner. First, we briefly review our current knowledge about the properties of two-headed motors. Next, we discuss the motor cycle underlying the 'hand-over-hand' motion of these molecules in Section 3. We provide a general theoretical framework for these motor cycles which we have recently applied to the specific case of kinesin. In Section 4, we describe the motor walks in the dilute regime of relatively low motor concentrations. In this regime, one can make rather robust estimates about the cargo transport in tube-like compartments and about the active cargo diffusion in slab-like compartments. In Section 5, cooperative transport of cargo by several motors will be discussed: both uni-directional transport by one species of motors and bi-directional transport arising from a 'tug-of-war' between two species of motors will be addressed. The last Section 6 summarizes our current understanding about crowding effects and phase transitions in motor traffic. A systematic study of crowding effects is likely to give us additional insight into the motors' behavior on the molecular scale. One example is provided by the concentration dependence of binding rates and bound state velocities which changes with the number of binding sites occupied by the motors.

\section{Two-headed motors: kinesins, dyneins, and myosins}

In the following, we will be primarily concerned with cytoskeletal motors that are processive in the sense that they are able to make many successive steps along a filament before they unbind from it. There are three superfamilies of processive motors that are found in essentially all eucaryotic cells [1,2]: kinesins, dyneins, and myosins.

Conventional kinesin (also known as kinesin-1) consists of two identical heavy chains which form two motor heads, see Fig. 2(a). Each of these heads has a catalytic domain, which adsorbs and hydrolyzes ATP, and another domain by which the motor head binds to the microtubule. The chemical free energy released from the hydrolysis of a single ATP molecule is transformed into a single step along the microtubule [28]. It is now well-established that kinesin walks in a 'hand-over-hand' fashion, i.e., by alternating steps in which one head moves forward while the other one remains bound to the tubule $[4,6]$. During one such step, the unbound head and the center-of-mass of the motor move by 16 and $8 \mathrm{~nm}$, respectively.

Conventional kinesin (and most other species of the kinesin superfamily) move towards the plus end of the microbule. Dynein motors, on the other hand, move in the opposite direction as indicated in Fig. 2(a). Dynein is also a two-headed motor consisting of two identical chains but has a more complex structure than kinesin; in particular, each head contains seven globular domains that are arranged in a ring-like manner [29]. Several of these domains can bind ATP and presumably hydrolyze it as well [30]. Dynein binds to the microtubule via a $13 \mathrm{~nm}$ long stalk [31]. There has been some evidence that the step size of dynein is variable and varies between 8 and $32 \mathrm{~nm}$ depending on load [32]. A recent study challenges this view and reports a step size of $8 \mathrm{~nm}$ independent of load [33]. Dynein seems to frequently switch between different protofilaments as it walks along the microtubule [34].

In addition to microtubules, actin filaments also provide tracks for motor molecules. Two types of myosins, $\mathrm{V}$ and VI, have been found to walk along these filaments in opposite directions as indicated in Fig. 2(b).

Myosin V consists of two heavy chains, each of which forms a motor head with a long neck or lever arm. Each lever arm contains six attached light chains as indicated by the globular domains in Fig. 2(b). Each head has a single catalytic domain for ATP hydrolysis [35]. Alternating hydrolysis events on the two heads also lead 
(a)

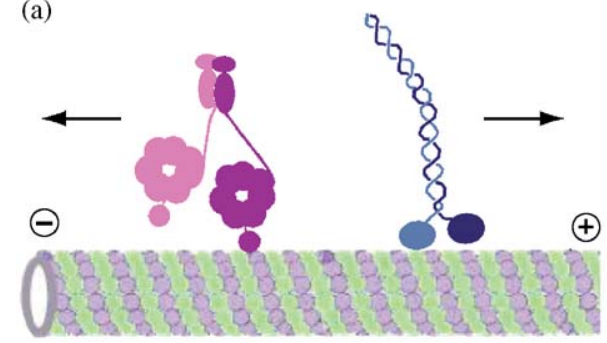

(b)

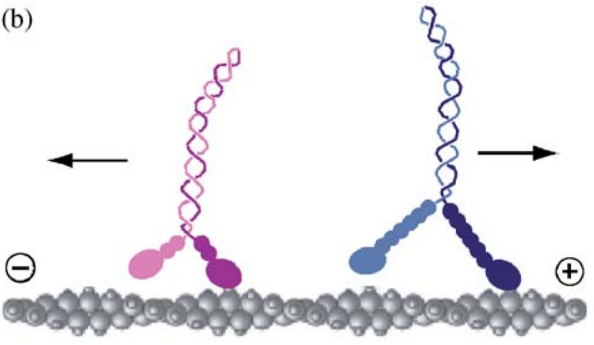

Fig. 2. Stepping motors that walk along cytoskeletal filaments, which are polar and have two different ends, a plus and a minus end: (a) kinesin and dynein motor that move to the plus and minus end, respectively, of a microtubule; and (b) myosin V and myosin VI that move to the plus (barbed) and minus (pointed) end, respectively, of an actin filament. The diameter of the microtubule and the actin filament are 25 and $8 \mathrm{~nm}$, respectively. For simplicity, the cargo binding domains of the motors have been omitted. All four types of molecular motors are dimers consisting of two identical protein chains and use ATP hydrolysis in order to move in a directed manner. Kinesin and the two myosin motors walk in a 'hand-over-hand' fashion. The step size of myosin VI is similar to the one of myosin V even though its lever arms are much shorter.

to 'hand-over-hand' motion [5] but with a step size of about $36 \mathrm{~nm}$ for the center-of-mass displacement [36] which corresponds to a displacement of about $72 \mathrm{~nm}$ for a single head. The $36 \mathrm{~nm}$ step size is close to the helical pitch of the actin filaments. This step size is modified for mutants with shortened lever arms [37]: Myosin V molecules with four light chains per lever arm are still processive but take only $24 \mathrm{~nm}$ steps; a further reduction to one light chain per lever arm leads to a nonprocessive motor with a $6 \mathrm{~nm}$ step size. In the absence of steric constraints, myosin V motors spiral around the filaments as they step forward [38].

Myosin V walks towards the plus or barbed end of the actin filament whereas myosin VI, another processive motor, walks towards its minus or pointed end, see Fig. 2(b). It has been argued that Myosin VI also walks 'hand over hand' along the actin filament [39]. Compared to myosin V, myosin VI has much shorter lever arms that involve only two light chains attached to each heavy chain as indicated in Fig. 2(b). Nevertheless, in the low load regime, it makes again steps of about $36 \mathrm{~nm}$ [40] which may indicate a stretchable element within the lever arms [39].

All four species of cytoskeletal motors displayed in Fig. 2 are two-headed and at least three of them walk in a 'hand-over-hand' manner. It has also been proposed that KIF1A kinesin is processive even in its monomeric state in which it has only a single motor head [41,42]. In general, such a motor could certainly exist and is also attractive from a theoretical point of view $[9,42]$. However, the case of KIF1A is somewhat controversial, since the monomers have a tendency to dimerize and the observed processivity could arise from this dimeric state [43].

\section{Motor cycles: chemistry plus mechanics}

Molecular motors have the ability to undergo a cyclic sequence of conformations or states. These states can differ in their chemical composition and/or in their spatial configuration. For the two-headed motors displayed in Fig. 2, each head can adsorb and hydrolyze ATP and, thus, attain several different chemical states. In addition, the motor transforms the chemical free energy released from this hydrolysis into mechanical work which represents a direct coupling between the chemical transitions and the mechanical steps.

When an ATP molecule is hydrolyzed by the catalytic domain of one motor head, one has, in principle, two different possibilites as far as the release of the hydrolysis products is concerned: the release of phosphate may precede the release of ADP or vice versa. For kinesin, several experimental studies indicate that phosphate is released first $[44,45]$. This implies that each head can be in three different chemical states: its catalytic domain may be empty (E), occupied by an ATP molecule (T), or occupied by an ADP molecule (D).

Since each head of kinesin exhibits three chemical states, the two-headed motor can attain $3^{2}=9$ such states as shown in Fig. 3(a). In this figure, the white heads are in the (E)-state, the black heads in the (T)-state, and the gray heads in the (D)-state. ATP adsorption leads to a transition from (E) to (T), ATP hydrolysis to a 

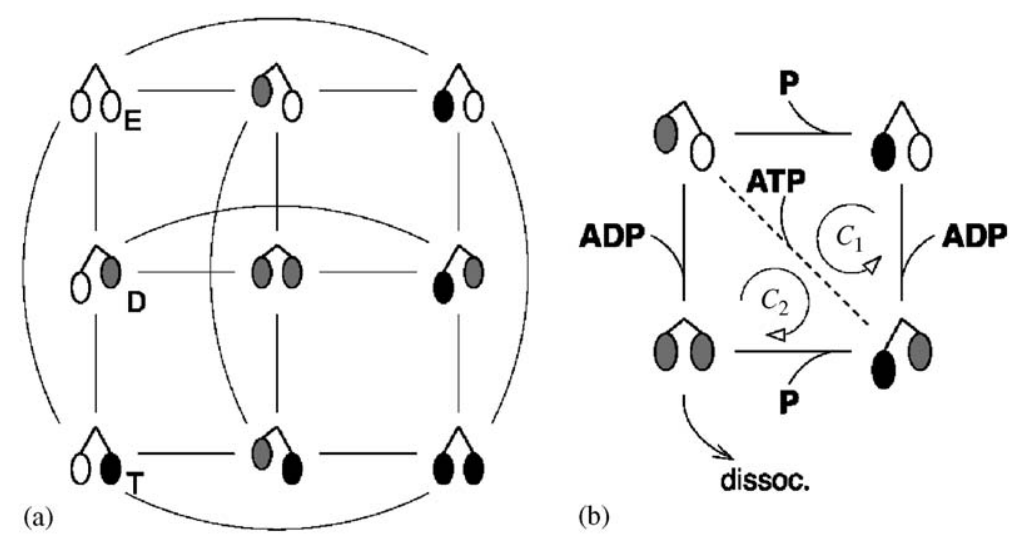

(b)

Fig. 3. (a) State space for a two-headed motor with $9=3^{2}$ different chemical states: each of the two heads can be empty (E, white), occupied by an ATP molecule (T, black), or by an ADP molecule (D, gray). This state space applies provided (i) each head contains a single catalytic domain for ATP hydrolysis and (ii) the release of phosphate from the head precedes the release of ADP. (b) For twoheaded kinesin, the state space can be reduced to four states consisting of two coupled chemomechanical cycles $\mathscr{C}_{1}$ and $\mathscr{C}_{2}$; the arrows indicate the direction of ATP-hydrolysis. The dashed line represents the transition at which the leading motor head, which was empty (white), binds ATP whereas the trailing head, which contains ADP (gray), undergoes a mechanical step and becomes the new leading head. For cycle $\mathscr{C}_{1}$, this transition is followed by ADP release from the new leading head. For cycle $\mathscr{C}_{2}$, the stepping transition is followed by ATP hydrolysis at the new trailing head.

transition from (T) to (D), and ADP release to a transition from (D) to (E). The motor head is taken to be strongly bound in its (T) and (E) states but to be weakly bound in its (D) state. Thus, the (DD) state in the middle of Fig. 3(a) represents the most weakly bound state from which the motor is most likely to unbind.

In order for the motor to be processive and, thus, to avoid the (DD) state, the chemical cycle on one head must be coordinated with the chemical cycle on the other head. An important ingredient in this coordination is that the binding of ATP on one head stimulates the ADP release on the other head [46]. This implies that one may focus on the three states in the upper right corner of Fig. 3(a) (or, equivalently, on the three states in the lower left corner of this figure). In this way, the state space is effectively reduced to three states of the motor cycle $\mathscr{C}_{1}$ shown in Fig. 3(b).

In order to obtain a complete description of the motor cycle, one must now correlate the transitions between the chemical states with the spatial displacements of the two motor heads. Recent experiments [47] give strong evidence that there are no mechanical substeps for kinesin, i.e., the mechanical step of the motor head occurs during a single transition of the $\mathscr{C}_{1}$-cycle shown in Fig. 3(b). A priori, one now has three possibilities: the trailing head could move forward (i) during ATP adsorption at the leading head, (ii) during ADP release from the trailing head, or (iii) during P release from the trailing head. These three possibilities lead to different motor properties. A detailed analysis of these three properties and a systematic comparison with the available experimental data leads to the conclusion that the trailing head moves forward as the leading head adsorbs ATP.

Our theoretical analysis is based on the time evolution of the probabilities $P_{i}(t)$ to find the motor in state $i$ at time $t$ which depends on the transition rates $\omega_{i j}$. These transition rates represent the number of transitions from state $i$ to state $j$ per unit time. The transition rates must satisfy two constraints arising from thermodynamics. First, in equilibrium, the rates must satisfy the detailed balance relation

$$
\prod_{\mathscr{C}} \frac{\omega_{i j}}{\omega_{j i}}=1 \text { for any motor cycle } \mathscr{C} \text {. }
$$

On the other hand, if the motor cycle $\mathscr{C}$ consumes the chemical energy $\Delta \mu_{\mathscr{C}}$ released by ATP hydrolysis and performs the work $W_{\mathscr{C}}$, the transition rates must satisfy the relation

$$
\ln \left(\prod_{\mathscr{C}} \frac{\omega_{i j}}{\omega_{j i}}\right)=\left(\Delta \mu_{\mathscr{C}}-W_{\mathscr{C}}\right) /\left(k_{B} T\right) .
$$


In addition, the relation between the fluxes and the transition rates exhibits some universal aspects which are useful in order to determine the functional dependence of these fluxes on a small number of rates $[27,48,49]$.

\section{Motor walks: directed and random}

The processive motors displayed in Fig. 2 can make many successive steps corresponding to many successive motor cycles. However, even if the motor's binding energy is large compared to the thermal energy $k_{B} T$, thermal fluctuations will eventually lead to an unbinding of the motor from the filament. Thus, every motor can be characterized by an average run length corresponding to an average number of steps along the filament. In the absence of load, kinesin motors bound to microtubules make about 100 successive steps which corresponds to an average run length of about $1 \mu \mathrm{m}$ [7]. In the absence and presence of dynactin, an accessory protein, dynein motors makes about 20 and 40 successive steps, respectively [50]. Myosin $\mathrm{V}$, which moves along actin filaments, makes about 50 steps before it unbinds again corresponding to a run length of about $1.5 \mu \mathrm{m}$ [51]. Myosin VI, on the other hand, makes only about 9 successive steps, i.e., its average run length is about $280 \mathrm{~nm}$ [39].

On length scales which are large compared to the run length, the motor undergoes peculiar walks which consist of directed movements along the filaments interrupted by periods of random, diffusive motion in the surrounding solution. In this section, we discuss these motor walks for the dilute regime of small motor concentration for which the mean separation of bound motors along the filament is large compared to their run length. In this dilute regime, interactions between the motor molecules can be safely ignored.

In order to be specific, let us again focus on kinesin which walks along microtubules. Each such microtubule consists of 13 protofilaments which provide a lattice of discrete binding sites for the kinesin motors. These binding sites correspond to the $\beta$-domain of the tubulin dimers [52] and are $8 \mathrm{~nm}$ apart. After a kinesin has bound to the microtubule, it walks along a single protofilament without sidesteps [53] in a 'hand-over-hand' manner [4,6] as schematically shown in Fig. 4(a). At each step, one head is displaced by $16 \mathrm{~nm}$ whereas the center-of-mass is displaced by $8 \mathrm{~nm}$.

Since we now want to describe the movement of the motor on length scales which are large compared to the step size $\ell=8 \mathrm{~nm}$, we introduce a reduced description and replace the motor molecule by a walker that makes discrete steps along the lattice of binding sites as shown in Fig. 4(b). Within one time step, the motor stays at the same site with probability $\gamma$, makes a forward step with probability $\alpha$, a backward step with probability $\beta$, and unbinds from the filament with unbinding probability $\varepsilon_{o}$. These four hopping probabilities can be uniquely related to the experimentally determined motor properties [27]. The average velocity of the bound motor, for example, is given by $v_{\mathrm{b}}=v_{\mathrm{b}, 0} \equiv\left(\ell / \tau_{\mathrm{b}}\right)(\alpha-\beta) /\left(1-\varepsilon_{o}\right)$, where $\ell$ is the step size and $\tau_{\mathrm{b}}$ an appropriate time scale as explained previously [27]. The notation $v_{\mathrm{b}, 0}$ refers to the motor's bound velocity in the absence of any additional forces or constraints acting on the walking motor. Likewise, the average run

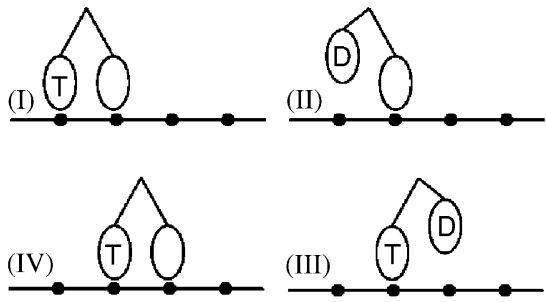

(a)

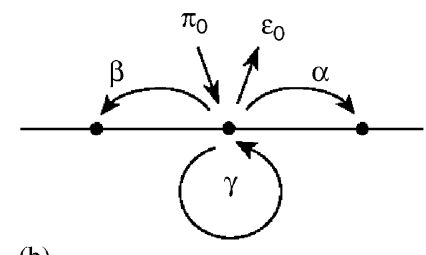

(b)

Fig. 4. (a) One forward step of two-headed kinesin along the lattice of binding sites provided by one protofilament. This step involves three conformations (I), (II), and (III) that differ in the chemical composition of their heads. As in Fig. 3, the catalytic site of each head can be empty (white), contain one ATP molecule (T), or one ADP molecule (D). The forward step occurs between conformation (II) and (III); and (b) reduction to a hopping process: within one time step, the motor stays at the same site with probability $\gamma$, makes a forward step to the next site on the right with probability $\alpha$, a backward step to the next site on the left with probability $\beta$, and unbinds from the filament with unbinding probability $\varepsilon_{o}$. In addition, when the unbound motor is close to a filament site, it can rebind to it with rebinding probability $\pi_{o}[9]$. 
length of the motor is given by $\Delta x_{\mathrm{b}}=\Delta x_{\mathrm{b}, 0}=\ell(\alpha-\beta) / \varepsilon_{o}$. For kinesin, backward steps are rather rare and $\beta \simeq 0$.

After the motor has detached from the filament, it will undergo undirected diffusive motion in the surrounding solution. From a theoretical point of view, it is convenient to describe this unbound motion of the motor as a random walk on a lattice as introduced in Ref. [9]. The properties of the motor walks can then be solved analytically for the simplest system geometries [54,55] and can be studied by mean field theory and simulations for more complex systems [9-11]. A lattice is, however, not essential and one can equally well study continuous diffusion in the unbound state as in Refs. [56,57]. When one of the motor heads of the diffusing molecule comes close to the filament, the motor will rebind to it with probability $\pi_{o}$ as indicated in Fig. 4(b). ${ }^{1}$

The binding probability $\pi_{o}$ can be related to measurable quantities as follows. Let us consider a system with volume $V$ that contains a certain number of filament binding sites $N_{\text {si }}$ and a certain number of motors $N_{\mathrm{mo}}=N_{\mathrm{b}}+N_{\mathrm{ub}}$, where $N_{\mathrm{b}}$ and $N_{\mathrm{ub}}$ represent the average number of bound and unbound motors, respectively. The bound motors are active which implies that their unbinding probability is given by $\varepsilon_{o}$ (which could differ from the unbinding probability in the absence of ATP). We then define the binding ratio $n_{\mathrm{b}} \equiv$ $N_{\mathrm{b}} / N_{\mathrm{si}}$ and the molar concentration $c \equiv N_{\mathrm{ub}} / N_{\mathrm{av}} V$ of the motors where $N_{\mathrm{av}}$ is Avogadro's number. In the limit of small concentration, the binding ratio behaves as $n_{\mathrm{b}} \approx c / K$ which defines the dissociation constant $K$. The latter constant has the dimension of a molar concentration and is experimentally found to be of $100 \mathrm{nM}$ for conventional kinesin.

On the other hand, if we describe the surrounding solution by a lattice with lattice parameter $\ell$, we may define the molar ratio of the unbound motors via $n_{\mathrm{ub}} \equiv \ell^{3} N_{\mathrm{av}} c$. The balance of binding and unbinding fluxes then leads to $\varepsilon_{o} n_{\mathrm{b}} \approx \pi_{o} n_{\mathrm{ub}}$ for small concentrations and, thus, to $\pi_{o}=\varepsilon_{o} / \ell^{3} N_{\mathrm{av}} K$. Note that $\pi_{o}$ is now expressed in terms of parameters which are all independent of the size and shape of the motor molecule. This independence applies, in fact, to all hopping probabilities and indicates that, in the limit of small motor concentrations, the description of the motor movement in terms of these hopping probabilities is rather general. Thus, in this limit, the same description also applies to a motor molecule which pulls a large cargo particle.

\subsection{Walks of single motors}

Now, consider the movement of a single motor in a slab-like geometry as shown in Fig. 5(a). The motor is initially placed onto the filament and will then undergo a motor walk consisting of directed movements interrupted by random, diffusive motion. On time scales that are large compared to the run time $\Delta x_{\mathrm{b}, 0} / v_{\mathrm{b}, 0}$, the probability $P_{\mathrm{b}}$ to find the motor bound to the filament will decay to zero for long times. On length scales that exceed the slab height, this decay is described by the power law $P_{\mathrm{b}}(t) \sim 1 / t^{\theta}$ with $\theta=\frac{1}{2}$. If the slab height is sufficiently large, the exponent $\theta$ will have the effective value $\theta=1$ up to a crossover time which is of the order of the motor's diffusion time across the slab. This implies that the motor moves, on average, with the longitudinal velocity [9]

$$
v_{\|} \sim v_{\mathrm{b}} P_{\mathrm{b}}(t) \sim v_{\mathrm{b}} / t^{\theta}
$$

parallel to the filament which also decays to zero with increasing time $t$.

\subsection{Motor walks in tube-like compartments}

Next, consider a tube-like compartment with length $L$ and cross-sectional area $A_{\text {cr. }}$ The compartment contains a dilute solution with $N_{\text {mo }}$ motors and several parallel filaments with $N_{\text {pr }}$ accessible protofilaments, as indicated in Fig. 5(b), which implies $N_{\mathrm{si}}=N_{\mathrm{pr}}(L / \ell)$ binding sites.

In order to suppress effects from the two ends of the tube, it is convenient to use periodic boundary conditions which could be experimentally realized by a torus-shaped tube. We then attain a steady state with

\footnotetext{
${ }^{1}$ The unbinding probability $\pi_{\mathrm{ad}}$ used in our previous work is related to $\pi_{o}$ via $\pi_{o}=n_{\mathrm{ad}} \pi_{\mathrm{ad}} / 6$, where $n_{\mathrm{ad}}$ is the number of nonfilament sites adjacent to a filament site.
} 


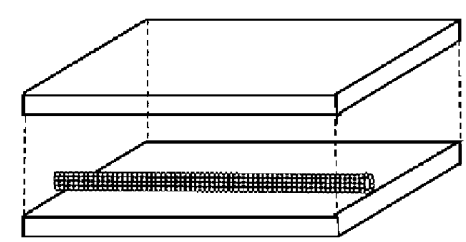

(a)

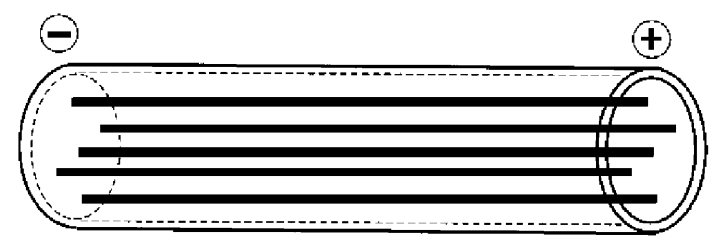

(b)

Fig. 5. (a) A slab-like compartment with a single filaments immobilized on the wall of the compartment; and (b) a tube-like compartment with several parallel filaments which all have the same orientation. The motors (not shown) undergo walks which consist of directed movements along the filaments interrupted by random, diffusive motion in the surrounding water.

an average number of bound motors, $N_{\mathrm{b}}$, and binding ratio $n_{\mathrm{b}}=N_{\mathrm{b}} / N_{\mathrm{si}} \approx c / K$ for small $c$. The mean separation of the bound motors is given by $\ell / n_{\mathrm{b}}$ which is large compared to the run length $\Delta x_{\mathrm{b}, 0}$ provided $c \ll K \ell / \Delta x_{\mathrm{b}, 0}$.

The bound state probability $P_{\mathrm{b}}$, which is defined as $P_{\mathrm{b}}=N_{\mathrm{b}} / N_{\mathrm{mo}}$, has the form

$$
P_{\mathrm{b}}=1 /\left(1+K \ell A_{\mathrm{cr}} / N_{\mathrm{pr}}\right) \text {. }
$$

Furthermore, in the dilute regime with $c \ll K \ell / \Delta x_{\mathrm{b}, 0}$, we can ignore any interaction effects between the bound motors and take the bound state velocity to have the zero force value $v_{\mathrm{b}}=v_{\mathrm{b}, 0}$. Therefore, the average velocity of a single motor parallel to the tube is now given by $[9,10]$

$$
v_{\|}=v_{\mathrm{b}} P_{\mathrm{b}}=v_{\mathrm{b}, 0} /\left(1+K \ell A_{\mathrm{cr}} / N_{\mathrm{pr}}\right)
$$

which explicitly shows the reduction of the motor displacement parallel to the filament by motor unbinding since the dissociation constant $K$ is proportional to the unbinding probability $\varepsilon_{o}$ or inversely proportional to the run length $\Delta x_{\mathrm{b}, 0}$. Therefore, the longitudinal motor velocity $v_{\|}$attains its maximal value $v_{\mathrm{b}, 0}$ in the limit of large run lengths. The motor flux $J$ through the tube is $J=J_{\mathrm{b}}=(\ell / L) N_{\mathrm{mo}} v_{\|}$which again increases with increasing run length.

\subsection{Active diffusion in slab-like compartments}

In general, one can use different types of filament patterns in order to induce different types of motor movements. In particular, one may also use slab-like geometries and filament patterns as shown in Fig. 6 in order to enhance the diffusion of the motors and their cargo. For the pattern in Fig. 6(a), several filaments with random orientation are located in each surface stripe. For the pattern in Fig. 6(b), all filaments have the same orientation within one surface stripe but neighboring stripes have opposite orientation. Such filament patterns can be constructed, e.g., by using striped surface domains or grooves covered by inactive motors or other chemical crosslinkers $[58-62,13,14]$. Another interesting method is provided by microfabricated surface pillars to which the filaments can be attached [63].

For the systems shown in Fig. 6, the motor and its cargo undergo nondirected but enhanced diffusion. The effective diffusion constant $D_{\text {eff }}$ is primarily determined by the competition of two length scales: the mesh size, say $L_{\perp}$, of the filament pattern and the average run length $\Delta x_{\mathrm{b}}$ of the motors [12]. If the mesh size exceeds the run length, i.e., for $\Delta x_{\mathrm{b}}<L_{\perp}$, the effective diffusion constant behaves as $D_{\text {eff }} \sim \Delta x_{\mathrm{b}} v_{\mathrm{b}}$. On the other hand, if the run length exceeds the mesh size, i.e., for $\Delta x_{\mathrm{b}}>L_{\perp}$, the diffusion constant is governed by the mesh size and one has $D_{\text {eff }} \sim L_{\perp} v_{\mathrm{b}}$ which corresponds to a random walk with effective step size $L_{\perp}$ and effective step time $L_{\perp} / v_{\mathrm{b}}$.

For a cargo particle in solution, the unbound diffusion constant is inversely proportional to the linear cargo size $R$ because of Stokes friction arising from the viscosity of the solution. In contrast, the effective diffusion constant for the active diffusion of molecular motors is hardly affected by the viscosity of the solution and, thus, is rather insensitive to the size of the cargo particle. Therefore, for micrometer-sized cargo particles in water, this effective diffusion constant for active diffusion can exceed the unbound diffusion constant by several orders of magnitude [12]. 

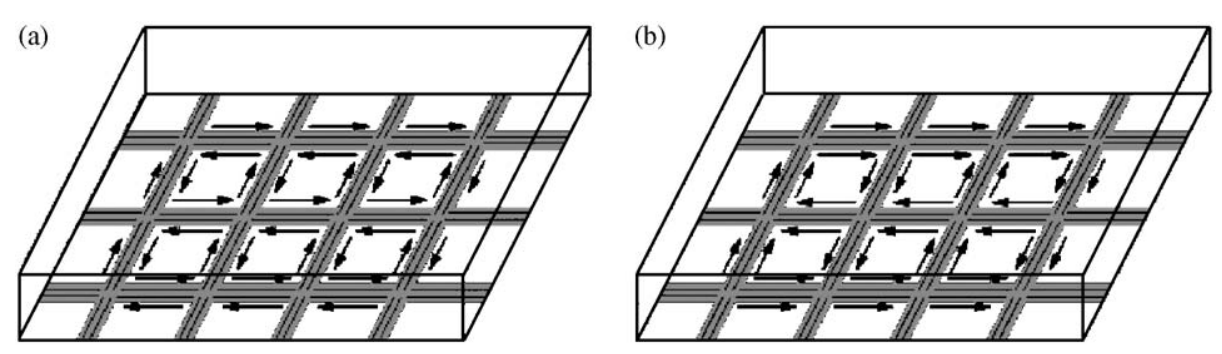

Fig. 6. Different patterns of filaments that lead to active and enhanced diffusion of motors: (a) striped surface domains, each of which contains immobilized filaments with both orientations; and (b) striped surface domains for which all filaments within one stripe have the same orientation but neighboring stripes have opposite orientation. The arrows indicate the direction of walking motors.

For a given type of motor, the maximal value for the effective diffusion constant $D_{\text {eff }}$ is $D_{\text {eff }} \sim \Delta x_{\mathrm{b}} v_{\mathrm{b}}$. Thus, in order to increase this coefficient, one must either increase the bound state velocity or the run length of the motors. One rather effective way to increase the run length is to use cooperative transport of cargo by several motors as discussed in the next section.

\section{Cargo transport by several motors}

In biological cells, molecular motors transport various types of cargo particles such as vesicles, organelles, and filaments. This cargo transport is typically performed by several motor molecules as revealed by electron microscopy [64,65] and single particle tracking [66-69].

One important consequence of this cooperative action is that it increases the run length of the cargo particle since this particle continues to move along the filament unless all motors unbind simultaneously. In addition, as long as the cargo particle is still connected to the filament by at least one motor, all unbound motors can rebind rather fast, because they are prevented from diffusing away from the filament. It has also been shown using in vitro motility assays that cargo particles pulled by many motors can switch tracks and move along several filaments at the same time, so that huge walking distances can be achieved which exceed the length of a single filament [14].

\subsection{Uni-directional transport by one motor species}

Now, consider a single cargo particle with $N$ motors which are firmly attached to the cargo, see Fig. 7 . When in contact with the filament, each motor has a finite run length and run time which implies that the actual number $n$ of pulling motors is not constant but varies with time between zero and $N$. Thus, if we include the unbound state with $n=0$, the cargo can be in $N+1$ different states $|n\rangle$ which are distinguished by the number $n$ of pulling motors.

If the cargo is in state $|n\rangle$, the binding of one motor to the filament leads to state $|n+1\rangle$ and the unbinding of one motor from the filament to state $|n-1\rangle$. If the motors do not interfere with each other, the corresponding transition rates, $\bar{\pi}_{n}$ and $\bar{\varepsilon}_{n}$, are proportional to the binding and unbinding probabilities, $\pi_{o}$ and $\varepsilon_{o}$, for a single motor as shown in Fig. 4(b). This leads to the evolution (or master) equation [8]

$$
\frac{\partial}{\partial t} P_{n}=\bar{\varepsilon}_{n+1} P_{n+1}+\bar{\pi}_{n-1} P_{n-1}-\left(\bar{\varepsilon}_{n}+\bar{\pi}_{n}\right) P_{n}
$$

for the probability $P_{n}$ to find the cargo in state $|n\rangle$ at time $t$. In the steady state, the probability distribution $P_{n}=P_{n}^{\text {st }}$ satisfies $\bar{\varepsilon}_{n+1} P_{n+1}^{\text {st }}=\bar{\pi}_{n} P_{n}^{\text {st }}$ for $0 \leqslant n \leqslant N-1$. This relation corresponds to detailed balance between the states $|n+1\rangle$ and $|n\rangle$ and reflects the fact that all movements of the bound cargo particle begin and end with $n=0$ and that every transition from $|n\rangle$ to $|n+1\rangle$ implies a backward transition at some later time. It is also implicitly assumed here that the binding and unbinding rates are independent of the transition rates for stepping along the filament. 

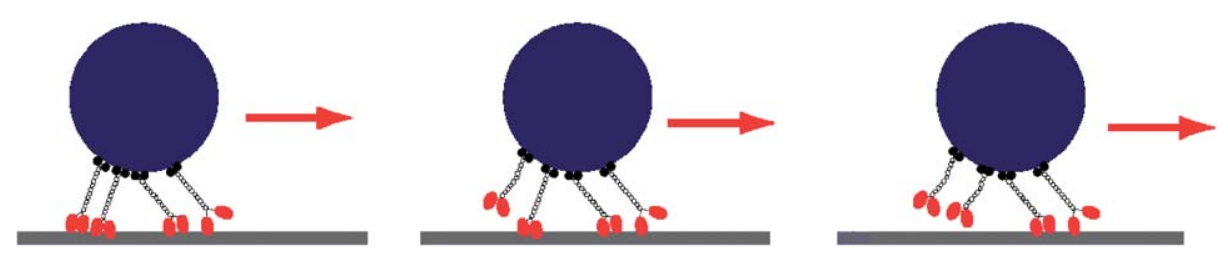

Fig. 7. A spherical cargo particle is transported cooperatively by $N$ molecular motors along a filament with $N=4$. The motors are firmly attached to the cargo but unbind from and rebind to the filament. Therefore, the actual number of pulling motors varies with time between 0 and $N$.

The steady-state probability distribution $P_{n}^{\text {st }}$ can be determined for arbitrary binding and unbinding rates [8]. If the motors do not interfere which each other, the average number $N_{\mathrm{b}}$ of bound motors is found to behave as $N_{\mathrm{b}} \approx N /(1+\kappa)$ for large $N$ with the desorption coefficient $\kappa \equiv \varepsilon_{o} / \pi_{o}$. Furthermore, if the bound velocity of the cargo is taken to be $v_{\mathrm{b}}$ for all bound states, a cargo with $N$ noninterfering motor molecules is characterized by the average run length

$$
\left\langle\Delta x_{\mathrm{b}}\right\rangle \approx\left(v_{\mathrm{b}} / \bar{\varepsilon}_{o} N\right)(1 / \kappa)^{N-1}
$$

for strongly binding motors with small $\kappa=\varepsilon_{o} / \pi_{o} \ll 1$ where $\bar{\varepsilon}_{o}$ is the unbinding rate of a single motor. Thus, in this case, the run length increases exponentially with increasing number of motors. If the cargo is pulled by up to $N$ kinesin motors, e.g., the run length is estimated to be $5^{N-1} / N$ micrometers which implies that seven or eight kinesin molecules are sufficient to attain an average walking distance in the centimeter range. For $N>1$, the run length distribution develops a fat tail [8] which is consistent with recent experiments by Beeg, Unger, and Dimova (private communication).

If the cargo particle is pulled against an external load force, this force is shared between the motors which provides a nontrivial motor-motor coupling and a generic mechanism for nonlinear force-velocity relationships. With increasing load force, the probability distribution of the instantaneous velocity is shifted towards smaller values, becomes broader, and develops several peaks [8] as recently observed for the in vivo transport of vesicles and organelles [67-69].

\subsection{Bi-directional transport by two motor species}

In biological cells, the motion of cargo particles along microtubules is often observed to be bi-directional in the sense that the particle frequently switches its direction of motion. Since both kinesin and dynein motors are bound to these particles, it is rather natural to assume that the bi-directional motion arises from the competition between these two motor species. Several proposals have been made for the molecular mechanisms underlying this competition; for recent reviews, see Refs. [70,71]. The presumably simplest mechanism consists in a 'tug-of-war' as shown in Fig. 8(a): there is a team of plus motors and a team of minus motors that pull in opposite directions, and the direction of motion is determined by the stronger team. However, since the number of motors that actually pull varies with time for both motor species, the weaker team may suddenly become the stronger one which reverses the direction of motion.

Such a 'tug-of-war' can be described by an appropriate generalization of the model as given by (6) to the case of two motor species. The cargo particle now carries $N_{+}$plus motors and $N_{-}$minus motors, and the cargo can attain $\left(N_{+}+1\right)\left(N_{-}+1\right)$ states $\left|n_{+}, n_{-}\right\rangle$which are characterized by the actual numbers $n_{+}$and $n_{-}$of plus and minus motors that pull at the same time. As soon as $n_{-}>0$, the plus motors experience a force arising from the minus motors and vice versa. Two sets of parameters turn out to be crucial for the behavior of this 'tug-of-war' model: (i) the desorption coefficients $\kappa_{+}$and $\kappa_{-}$, which govern the unbinding rates in the absence of force, and (ii) the ratios $f_{+}$and $f_{-}$between the stall and detachment forces which describe the competition between the two motors.

A particularly instructive case is obtained for $N_{+}=N_{-}, \kappa_{+}=\kappa_{-} \equiv \kappa$ and $f_{+}=f_{-} \equiv f$. The corresponding bifurcation diagram is shown in Fig. $8(\mathrm{~b})$ for $N_{+}=N_{-}=5$. This diagram exhibits three different regions denoted by $1 \Phi, 2 \Phi$, and $3 \Phi$ which correspond to three qualitatively different steady-state distributions 


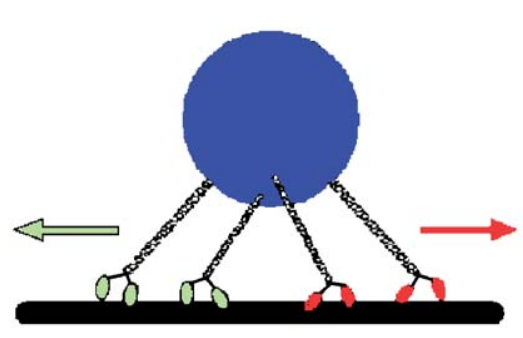

(a)

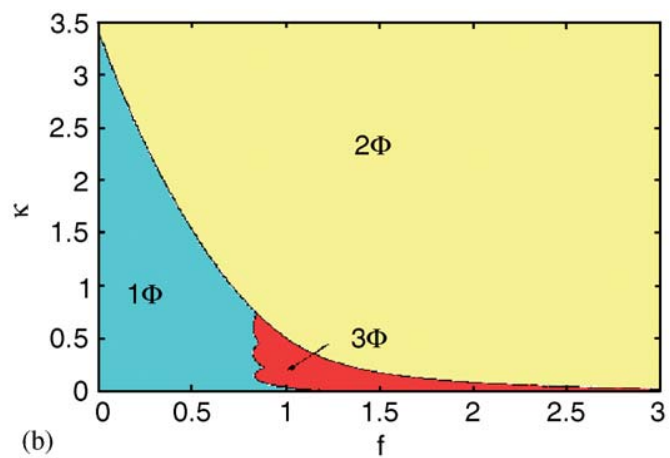

Fig. 8. (a) 'Tug of war' between two motor species that try to pull the cargo in opposite directions. In general, the cargo particle carries $N_{+}$ plus motors and $N_{-}$minus motors which are also the maximal numbers of plus and minus motors that can actually pull on the particle; and (b) bifurcation diagram for $N_{+}=N_{-}=5$. For simplicity, all motors are taken to have the same desorption coefficient $\kappa=\varepsilon_{o} / \pi_{o}$ and the same ratio $f=F_{S} / F_{d}$ of stall force versus detachment force. The bifurcation diagram exhibits three regions $1 \Phi$ (blue), $2 \Phi($ yellow), and $3 \Phi$ (red) which are distinguished by qualitatively different motor number distributions with one, two, and three maxima, respectively, as explained in the text.

$P\left(n_{+}, n_{-}\right)$for the actual motor numbers. These distributions have one, two, or three maxima representing one, two, or three different motility states arising in the 'tug-of-war'.

In the region denoted by $1 \Phi$, which is located at small $\kappa$ and small $f$, the steady-state distribution has only one maximum corresponding to a single motility state for which the order parameter $\Delta \equiv n_{+}-n_{-}$vanishes. This state corresponds to a cargo particle that is characterized by zero average velocity and, thus, vanishing motility. In region $2 \Phi$, the distribution has two maxima which correspond to a majority of plus and minus motors and represent two competing motility states in which the cargo particle moves into the plus or minus direction, respectively. Finally, in region $3 \Phi$, which is located at small $\kappa$ but large $f$, all three possible motility states compete. The time evolution of the distributions strongly indicates that these competing states become separate nonequilibrium phases in the limit of large $N_{+} \sim N_{-}$.

\section{Motor traffic: jams and more}

In order to simplify the following discussion, we will now use the term 'motor particle' in order to collectively refer to (i) single molecular motors and to (ii) single cargo particles which carry one or several motors. So far, we have discussed the movements of motor particles in the dilute regime for which the mean separation of the bound motor particles is large compared to their average run length. As we increase the concentration of these motor particles, these particles will start to interact with one another which leads to jamming of motors and crowding of filaments, to nonuniform density and flux patterns of motor traffic, and to traffic phase transitions.

\subsection{Crowding by processive motors}

The simplest theoretical framework by which one can study interacting motor particles in a systematic manner is again provided by lattice models as described in Section 4 for the regime of low motor concentration. The most basic interaction that all motor particles experience is mutual exclusion from a given spatial location. In order to incorporate these short-ranged repulsive interactions, the motor particles are now taken to occupy a certain lattice volume $[9,27]$.

In general, the local density of the motors will be nonuniform both along the filaments and in the surrounding solution [10]. However, in many cases, the solution acts as a large reservoir of motors, and the density gradients of the unbound motors are rather small. In these cases, we may ignore possible depletion layers of the unbound motors close to the filaments and describe the unbound motors by their volume fraction or molar concentration. In this limit, the balance of fluxes perpendicular to the filament simplifies and reduces to the equilibrium between binding and unbinding fluxes [10]. 


\subsubsection{One site occupancy of motors}

Since the motors bind to the filaments via their motor heads and since the remaining parts of the motor molecules are rather flexible, one may focus on the mutual exclusion of the heads close to the filament. Thus, the volume excluded by a motor molecule at a single filament site will be at least as large as the volume of one motor head. For kinesin, the motor head is a globular structure and has a volume which is of the order of $\ell^{3}$ where $\ell$ is the lattice parameter of the microtubule.

If we further assume, that the bound motor is connected to the filament by a single head during most of its motor cycle, the whole motor particle will occupy a single filament site as well. In this case, the binding ratio $n_{\mathrm{b}}$ is equal to the fraction $\rho_{\mathrm{b}}$ of occupied binding sites. The mutual exclusion of the motors further away from the filament depends on the detailed geometry of the motor molecule and its cargo and will affect the relation between the molar ratio $n_{\mathrm{ub}}$ of the unbound motors and their volume fraction $\rho_{\mathrm{ub}}$. If one ignores the difference between the molar ratio and the volume fraction, one obtains the simple balance equation $\bar{\varepsilon}_{o} \rho_{\mathrm{b}}=\bar{\pi}_{o} \rho_{\mathrm{ub}}\left(1-\rho_{\mathrm{b}}\right)$ for the unbinding and binding fluxes as in Ref. [10]. On the other hand, the relation $\rho_{\mathrm{b}}=n_{\mathrm{b}} \approx c / K$, which defines the dissociation constant $K$ in the limit of low motor concentration, also implies that

$$
\bar{\varepsilon}_{o} \rho_{\mathrm{b}}=\left(\bar{\varepsilon}_{o} / K\right) c\left(1-\rho_{\mathrm{b}}\right) \equiv \bar{\chi} c
$$

which defines the effective binding rate $\bar{\chi}$ (with the dimension of an inverse molar concentration per time). Solving this equation for the fraction $\rho_{\mathrm{b}}$ of occupied binding sites, one obtains the Langmuir-type equation $\rho_{\mathrm{b}}=c /(K+c)$ and, thus, the effective binding rate

$$
\bar{\chi}=\bar{\chi}(c)=\bar{\varepsilon}_{o} /(K+c) \text {. }
$$

In addition, the bound flux along a single filament is given by $J_{\mathrm{b}}=v_{\mathrm{b}, 0} \rho_{\mathrm{b}}\left(1-\rho_{\mathrm{b}}\right)$ [10] which is valid irrespective of the size and shape of the motor as long as each motor occupies only one binding site, i.e., as long as $\rho_{\mathrm{b}}=n_{\mathrm{b}}$. The effective bound velocity $v_{\mathrm{b}}$ now follows from $J_{\mathrm{b}}=n_{\mathrm{b}} v_{\mathrm{b}}=\rho_{\mathrm{b}} v_{\mathrm{b}}$ which leads to

$$
v_{\mathrm{b}}=v_{\mathrm{b}}(c)=v_{\mathrm{b}, 0} K /(K+c) .
$$

This is the average velocity of the bound motor which is reduced compared to $v_{\mathrm{b}, 0}$ as a result of the crowded filament. Note that, for one site occupancy of the motor as discussed here, both the effective binding rate $\bar{\chi}$ and the average bound velocity $v_{\mathrm{b}}$ have the same dependence on the motor concentration $c$.

\subsubsection{Two site occupancy of motors}

Two-headed motors such as kinesin, which move in a 'hand-over-hand' fashion, must be bound to the filament with both heads for some fraction of their motor cycle, compare Fig. 4. Thus, let us now assume that the motors bind with both heads and, thus, occupy two neighboring binding sites during most of their motor cycle which agrees with the conclusion from recent single molecule experiments [6]. The adsorption of particles that occupy several binding sites has been previously addressed in the context of ligand-binding to DNA [72]. On the other hand, the flux of such particles, which are irreversibly bound to a one-dimensional track, has also been studied as a model for ribosome movement along RNA [73]. If one extends these considerations to the motor/filament systems discussed here, one finds that a motor that occupies two binding sites has the effective binding rate

$$
\bar{\chi}=\bar{\chi}(c)=2 \bar{\varepsilon}_{o} /(K+4 c+\sqrt{K(K+4 c)})
$$

and the average bound velocity

$$
v_{\mathrm{b}}=v_{\mathrm{b}}(c)=v_{\mathrm{b}, 0} 2 \sqrt{K} /(\sqrt{K+4 c}+\sqrt{K}) .
$$

The dissociation constant $K$ is still defined via the binding ratio $n_{\mathrm{b}}$ which behaves as $n_{\mathrm{b}} \approx c / K$ for small motor concentration but one now has $n_{\mathrm{b}}=\rho_{\mathrm{b}} / 2$.

Thus, if the bound motors occupy two binding sites, the concentration dependence of the effective binding rate $\bar{\chi}$ and the effective velocity $v_{\mathrm{b}}$ are quite different. In fact, a combination of the two relations (11) and (12) leads to

$$
\frac{\bar{\chi}}{v_{\mathrm{b}}}=\frac{\bar{\varepsilon}_{o}}{v_{\mathrm{b}, 0} \sqrt{K}} \frac{1}{\sqrt{K+4 c}}
$$


which explicitly shows that the effective binding rate decreases faster than the effective velocity with increasing motor concentration $c$.

In recent experiments by Seitz and Surrey [20], both the effective binding rate $\bar{\chi}$ and the average velocity $v_{\mathrm{b}}$ of the bound motors have been measured for conventional kinesin as a function of the motor concentration $c$. It was indeed observed that the velocity decays more slowly with $c$ than the effective binding rate. In addition, the binding ratio $n_{\mathrm{b}}$ was measured as a function of motor concentration $c$ (i) for kinesins that walked in the presence of ATP and (ii) for statically bound kinesin in the presence of the nonhydrolyzable ATP analogue AMP-PNP. As the motor concentration is increased, the binding ratio increases in both cases but this increase is strongly reduced for the walking kinesins. However, it is difficult to decide on the basis of the available data if the binding ratio $n_{\mathrm{b}}$ has already saturated for the largest concentrations that were experimentally accessible in Ref. [20]. If one assumes such a saturation, the saturation value $n_{\mathrm{b}, *}^{\mathrm{wa}}$ for the walking kinesins is reduced by about a factor two compared to the saturation $n_{\mathrm{b}, *}^{\mathrm{st}}$ of the statically bound kinesins.

Such a reduction in the saturation value of the binding ratio would imply that, under the conditions used in Ref. [20], static kinesins occupy, on average, only half as much space on the filaments as moving kinesins. If static kinesins occupied only one binding site, the two site occupancy of walking kinesins would lead to the concentration dependencies for the binding rate and bound motor velocity as given by (11) and (12). As mentioned, these latter relationships are in qualitative agreement with the data in [20] since the velocity was found to decay more slowly with increasing motor concentration than the effective binding rate.

On the other hand, decoration experiments indicate that static kinesins occupy at least 1.3 binding sites [74]. The data in Ref. [20] would then imply that walking kinesins occupy at least 2.6 binding sites. This increased site occupancy of walking kinesins could arise from various molecular mechanisms: (i) they could increase the effective lateral size of their motor domains, e.g., by some conformational change in the neck region. However, this would be difficult to reconcile with the single molecule data in Ref. [6]; (ii) they could bump into each other and, in this way, increase the unbinding rate and reduce the binding rate. However, an increased unbinding rate has not been observed in Ref. [20] (Surrey, private communication); (iii) they could also reduce the binding rate by hydrodynamic interactions which would be effective even if the motors made no direct molecular contacts; or (iv) they could assume a certain conformation that acts as a spacer between adjacent walkers. Further experiments should enable us to distinguish between these possible mechanisms.

\subsection{Crowding by another molecular species}

Crowding effects will also arise if the system contains another molecular species which binds to the same filaments as the motors and which is sufficiently large to exclude the motors from their binding sites on these filaments. This happens, for example, if the system contains another species of processive motors which walk in the opposite direction as has been theoretically studied in Ref. [26]. Another example is provided by nonprocessive motors that act as obstacles for the processive ones. The latter system has been experimentally studied in Ref. [20] where conventional kinesins competed with nonprocessive E164A kinesin mutants for the same binding sites. These kinesin mutants bind to microtubules for about $200 \mathrm{~ms}$ and then unbind again via a single 'step'.

In general, for each type of processive motor, one should distinguish two types of obstacles depending on the binding time of these obstacles. Strongly bound obstacles have a binding time that is large compared to the average run time of the processive motors and that remains essentially unaffected by collisions with the processive motors. Weakly bound obstacles, on the other hand, have a binding time that is comparable with or smaller than this average run time. The E164A kinesin mutants represent weakly bound obstacles since their binding time of about $200 \mathrm{~ms}$ is smaller than the average run time of conventional kinesin which is of the order of $1 \mathrm{~s}$.

As an example, consider a tube-like compartment as in Section 4 which contains a low concentration $c$ of processive motors and a larger concentration $c_{2}$ of the second molecular species which provides weakly bound obstacles. The processive motors have the dissociation constant $K$ as before and make only forward steps with probability $\alpha$. The dissociation constant of the obstacles is denoted by $K_{2}$. A single obstacle, which is bound to a filament site and is not pushed by a processive motor, is characterized by the dwell probability $\gamma_{2}$ and the unbinding probability $\varepsilon_{o, 2}=1-\gamma_{2}$. If a processive motor is located directly in front of the obstacle, this 
obstacle is taken to unbind with the increased probability $\zeta \varepsilon_{o, 2}$ with $\zeta>1$ which describes active unbinding of the obstacle by the forward stepping motor.

For simplicity, let us assume that both motor and obstacle occupy a single binding site. The binding ratio $n_{\mathrm{b}, 2}$ of the obstacles is then equal to the fraction $\rho_{\mathrm{b}, 2}$ of binding sites occupied by the obstacles with $\rho_{\mathrm{b}, 2}=c_{2} /\left(K_{2}+c_{2}\right)$. For low concentrations $c$ of the processive motors, the balance of the binding and unbinding fluxes now leads to the effective binding rate

$$
\bar{\chi}=\bar{\chi}\left(c_{2}\right) \approx \frac{\bar{\varepsilon}_{o}}{K} \frac{K_{2}}{K_{2}+c_{2}}
$$

which describes the exclusion of the motors by the obstacles. Likewise, the bound processive motor now has the average velocity

$$
v_{\mathrm{b}}=v_{\mathrm{b}}\left(c_{2}\right)=v_{\mathrm{b}, 0} \frac{K_{2}}{K_{2}+c_{2} / \zeta+\tilde{\rho}_{\mathrm{b}, 2} / \zeta}
$$

with $\tilde{\rho}_{\mathrm{b}, 2} \equiv\left(\alpha K_{2} / \varepsilon_{o, 2}\right) \rho_{\mathrm{b}, 2}$. For $\zeta=1$, i.e., if there is no active unbinding of the obstacles by the motors, the velocity $v_{\mathrm{b}}$ of the bound motors decays somewhat faster than the effective binding rate $\bar{\chi}$ with increasing obstacle concentration $c_{2}$. However, for $\zeta \gg 1$, i.e., for strong active unbinding, one recovers the velocity $v_{\mathrm{b}, 0}$ of the bound motor in the absence of obstacles.

\subsection{Additional aspects of cooperative motor behavior}

In the previous subsections, we have discussed one aspect of cooperative motor behavior, namely traffic jams. From a theoretical point of view, such jams are rather natural traffic phenomena for molecular motors [9] but, in the case of conventional kinesin, the experimental search for such jams has led to some rather interesting observations [20] which remain to be clarified.

Theoretically, traffic jams are most conveniently studied for ring-like arrangements of the oriented filaments corresponding to periodic boundary conditions. In this case, the steady state has a nonzero motor flux but both the bound motor density and the bound motor flux are spatially uniform. Spatially nonuniform patterns of motor densities and fluxes can be induced in tube-like compartments by enforcing different types of boundary conditions. Closed boundary conditions, for example, lead to a partitioning of the tube into a rather crowded and a nearly empty tube segment and, thus, to an actively formed interface [9].

For an open tube with controlled boundary densities at the two ends, one obtains three different phases in close analogy to the so-called asymmetric simple exclusion process (ASEP) in one dimension as we first pointed out in Ref. [10]. Other groups have studied one-dimensional ASEPs coupled to particle reservoirs but with system-size dependent unbinding and binding rates [22-24]. Since the phase transitions in open tubes are controlled by the boundary densities, they are difficult to study experimentally.

As far as the motor traffic is concerned, the geometry of an axon as shown in Fig. 1 corresponds to a halfopen tube, which is coupled to a motor reservoir at one end but is closed at the other end [25]. The closed end corresponds to the synaptic terminal whereas the motor reservoir at the open end corresponds to the cell body, where the motors are synthesized. As previously mentioned, kinesin motors walk to the closed end, and dynein motors walk to the open end. If dyneins are released at the open end, they can diffuse into the tube until they are bound to the filament and then walk back to the open end. In the steady state, the diffusive and bound motor fluxes balance, and the bound dyneins form a jam at the open end of the tube with a jam length that diverges as $\sim 1 / v_{\mathrm{b}}$ for small velocity $v_{\mathrm{b}}$ of the bound motor [25]. The latter singularity can be explored by controlling the ATP concentration.

Another type of transition, which can be explored by changing the motor concentrations, occurs in systems with two species of motors that walk in opposite directions. The simplest geometry consists in a ring-like filament arrangement which is in contact with a bulk reservoir for the two motor species. Alternatively, one may consider a tube-like compartment with periodic boundary conditions as shown in Fig. 9. As long as the motor-motor interactions are purely repulsive, the flux of bound motors is determined by the majority species and the system evolves smoothly as one varies the motor concentrations. Decoration experiments indicate, however, that motors of the same species bind to the filament in a correlated fashion, i.e., a plus motor is more 


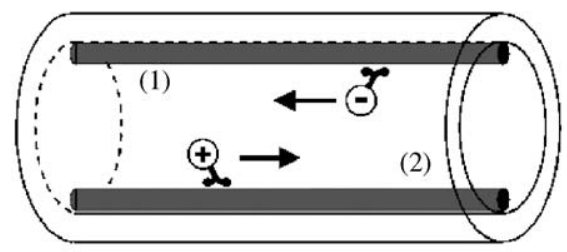

(a)

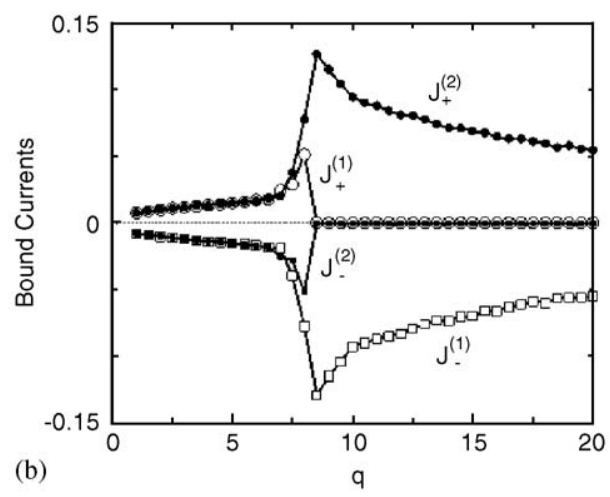

Fig. 9. (a) Tubular compartment with two parallel filaments (1) and (2), which have the same orientation, and with an equal number of plus and minus motor particles. For interaction parameter $q>q_{c}$, the symmetry of the system is spontaneously broken and one obtains a quasi-steady state in which one filament is occupied by plus motor particles, the other by minus ones. (b) Different bound currents for the steady state shown in (a) as a function of the interaction parameter $q$. The plus motor particles generate the bound currents $J_{+}^{(1)}$ and $J_{+}^{(2)}$ on filament (1) and (2), respectively; likewise, the minus motor particles generate the currents $J_{-}^{(1)}$ and $J_{-}^{(2)}$. Above the critical value $q=q_{c}$ of the interaction parameter, both types of motors walk on both filaments.

likely to bind close to another plus motor [75-78]. If one describes this effectively attractive motor-motor interaction via an interaction parameter $q$, the systems undergoes a traffic phase transition if $q$ exceeds a critical value $q_{\mathrm{c}}$ [26]. This phase transition occurs between two states with a spontaneously broken symmetry, where one motor species is essentially excluded from the filaments. As one varies the bulk composition of the motors, the total motor flux develops a hysteresis loop across the phase boundary. In addition, if the system contains several filaments, the broken symmetry is directly visible via the coexistence of traffic lanes with opposite directionality as illustrated in Fig. 9.

\section{Summary and outlook}

Molecular motors that walk along cytoskeletal filaments - microtubules or actin filaments - exhibit several motility regimes that cover many length and time scales. On the subnano scale, the motor heads undergo cyclic sequences of conformational transitions or motor cycles which transform the chemical energy of ATP into mechanical steps. For kinesin, the available data are consistent with two coupled motor cycles as shown in Fig. 3(b). Kinesin is able to perform about 100 successive motor cycles and in this way make about 100 forward steps, as illustrated in Fig. 4(a), before it unbinds from the filament. The corresponding run length is about $1 \mu \mathrm{m}$.

On length scales which are large compared to the run length, the motor undergoes peculiar walks that consist of directed movements along the filaments interrupted by random, diffusive motion in the surrounding water. These walks can be used for cargo transport through tube-like compartments as shown in Fig. 5(b). The flux of cargo can be enhanced by larger run lengths. One rather efficient way to increase the run length is to attach several molecular motors to the cargo. Indeed, the average run length increases exponentially with the number $N$ of attached motors as described by relation (7).

In biological cells, vesicles and other cargo particles often carry both kinesins and dyneins which leads to bidirectional transport along microtubules. The presumably simplest mechanism for this kind of movement is provided by the competition or 'tug-of-war' between the two motor species. As described in Section 5.2, this competition leads to motor number distributions with one, two, or three maxima corresponding to coexisting 'states' which differ in their motility. The dependence of these 'motility states' on the parameters of the system can be summarized in bifurcation diagrams as shown in Fig. 8(b).

As one increases the concentration of the motors, the filaments start to become crowded, and the transport flux is predicted to be reduced by traffic jams. The experimental study of such jams has only begun rather recently and led to some interesting observations which remain to be clarified. Depending on the system geometry and on the filament arrangements, the motor traffic can assume various density and flux patterns 
and can undergo phase transitions between these pattern. One example is provided by the spontaneous symmetry breaking in systems with two motor species as illustrated in Fig. 9.

Biomimetic transport systems based on molecular motors represent promising alternatives to microfluidic devices in which transport is coupled to flow induced by external pressure. Compared to pressure-induced flow, the transport by motors has several advantages such as: (i) cargo transport is hardly affected by the viscosity of the aqueous solution and, thus, remains efficient even in a dense solution of macromolecules; (ii) using two different motor species, different types of cargo can be simultaneously transported in both directions; and (iii) this transport system does not require rigid compartment walls but works in soft and flexible compartments as well. Another application of motors is their active diffusion by which one can increase the diffusion constant of micrometer-sized cargo particles by several orders of magnitude as explained in Section 4.3. When integrated into existing biochips for DNA and RNA hybridization, these transport systems would act to increase the hybridization rates.

In general, active biomimetic systems based on molecular motors and filaments have many potential applications in bioengineering, pharmacology and medicine. Such applications include sorting devices for biomolecules, motile drug delivery systems, molecular shuttles in 'labs-on-a-chip', and switchable scaffolds for tissue engineering. Thus, molecular motors and filaments are likely to become key components in bionanotechnology.

\section{References}

[1] M. Schliwa, G. Woehlke, Molecular motors, Nature 422 (2003) 759-765.

[2] R.D. Vale, The molecular motor toolbox for intracellular transport, Cell 112 (2003) 467-480.

[3] J. Howard, Mechanics of Motor Proteins and the Cytoskeleton, first ed., Sinauer, New York, 2001.

[4] K. Svoboda, Ch.F. Schmidt, B.J. Schnapp, St.M. Block, Direct observation of kinesin stepping by optical trapping interferometry, Nature 365 (1993) 721-727.

[5] A. Yildiz, J.N. Forkey, S.A. McKinney, T. Ha, Y.E. Goldmann, P.R. Selvin, Myosin V walks handover-hand: single fluorophore imaging with 1.5-nm localization, Science 300 (2003) 2061-2065.

[6] A. Yildiz, M. Tomishige, R.D. Vale, P.R. Selvin, Kinesin walks hand-over-hand, Science 303 (2004) 676-678.

[7] St.M. Block, L.S.B. Goldstein, B.J. Schnapp, Bead movement by single kinesin molecules studied with optical tweezers, Nature 348 (1990) 348-352.

[8] S. Klumpp, R. Lipowsky, Cooperative cargo transport by several molecular motors, Proc. Nat. Acad. Sci. USA 102 (2005) 17284-17289.

[9] R. Lipowsky, S. Klumpp, T.M. Nieuwenhuizen, Random walks of cytoskeletal motors in open and closed compartments, Phys. Rev. Lett. 87 (2001) 108101.

[10] S. Klumpp, R. Lipowsky, Traffic of molecular motors through open tube-like compartments, J. Stat. Phys. 113 (2003) 233-268.

[11] S. Klumpp, T.M. Nieuwenhuizen, R. Lipowsky, Self-organized density patterns of molecular motors in arrays of cytoskeletal filaments, Biophys. J. 88 (2005) 3118-3132.

[12] S. Klumpp, R. Lipowsky, Active diffusion of motor particles, Phys. Rev. Lett. 95 (2005) 268102.

[13] H. Hess, J. Clemmens, D. Qin, J. Howard, V. Vogel, Light-controlled molecular shuttles made from motor proteins carrying cargo on engineered surfaces, Nano Lett. 1 (2001) 235-239.

[14] K.J. Böhm, R. Stracke, P. Mühling, E. Unger, Motor protein-driven unidirectional transport of micrometer-sized cargoes across isopolar microtubule arrays, Nanotechnology 12 (2001) 238-244.

[15] S. Konzack, Funktion des kinesin motorproteins KipA bei der organisation des mikrotubuli-cytoskeletts und beim polaren wachstum von Aspergillus nidulans, Doctoral Thesis, University of Marburg, 2004.

[16] S. Konzack, P.E. Rischitor, C. Enke, R. Fischer, The role of the kinesin motor KipA in microtubule organization and polarized growth of Aspergillus nidulans, Mol. Biol. Cell 16 (2005) 497-506.

[17] D.D. Hurd, W.M. Saxton, Kinesin mutations cause motor neuron deisease phenotypes by disrupting fast axonal transport in drosophila, Genetics 144 (1996) 1075-1085.

[18] M.A. Martin, S.J. Iyadurai, A. Gassman Jr., J.G. Gindhart, T.S. Hays, W.M. Saxton, Cytoplasmic dynein, the dynactin complex, and kinesin are interdependent and essential for fast axonal transport, Mol. Biol. Cell 10 (1999) 3717-3728.

[19] L.S.B. Goldstein, Kinesin molecular motors: transport pathways, receptors, and human disease, Proc. Nat. Acad. Sci. USA 98 (2001) 6999-7003.

[20] A. Seitz, T. Surrey, Processive movement of single kinesins on crowded microtubules visualized using quantum dots, EMBO J. 25 (2006) 267-277.

[21] S. Klumpp, R. Lipowsky, Asymmetric simple exclusion processes with diffusive bottlenecks, Phys. Rev. E 70 (2004) 066104.

[22] A. Parmeggiani, T. Franosch, E. Frey, Phase coexistence in driven one-dimensional transport, Phys. Rev. Lett. 90 (2003) 086601. 
[23] V. Popkov, A. Rakos, R.D. Willmann, A.B. Kolomeisky, G.M. Schütz, Localization of shocks in driven diffusive systems without particle number conservation, Phys. Rev. E 67 (2003) 066117.

[24] M.R. Evans, R. Juhasz, L. Santen, Shock formation in an exclusion process with creation and annihilation, Phys. Rev. E 68 (2003) 026117.

[25] M. Müller, S. Klumpp, R. Lipowsky, Molecular motor traffic in a half open tube, J. Phys.: Condens. Mater 17 (2005) S3839-S3850.

[26] S. Klumpp, R. Lipowsky, Phase transitions in systems with two species of molecular motors, Europhys. Lett. 66 (2004) 90-96.

[27] R. Lipowsky, S. Klumpp, Life is motion-multiscale motility of molecular motors, Physica A 352 (2005) 53-112.

[28] M.J. Schnitzer, S.M. Block, Kinesin hydrolyses one ATP per $8 \mathrm{~nm}$ step, Nature 388 (1997) 386-390.

[29] M. Samsó, M.P. Koonce, 25 A resolution structure of a cytoplasmic dynein motor reveals a seven-member planar ring, J. Mol. Biol. 340 (2004) 1059-1072.

[30] R. Mallik, S.P. Gross, Molecular motors: strategies to get along, Curr. Biol. 14 (2004) R971-R982.

[31] M.A. Gee, J.E. Heuser, R.B. Vallee, An extended microtubule-binding structure within the dynein motor domain, Nature 390 (1997) 636-639.

[32] R. Mallik, B.C. Carter, S.A. Lex, S.J. King, S.P. Gross, Cytoplasmic dynein functions as a gear in response to load, Nature 427 (2004) 649-652.

[33] S. Toba, T.M. Watanabe, L. Yamaguchi-Okimoto, Y.Y. Toyoshima, H. Higuchi, Overlapping hand-over-hand mechanism of single molecular motility of cytoplasmic dynein, Proc. Nat. Acad. Sci. USA 103 (2006) 5741-5745.

[34] Z. Wang, S. Khan, M. Sheetz, Single cytoplasmic dynein molecule movements, characterization and comparison with kinesin, Biophys. J. 69 (1995) 2011-2023.

[35] R.D. Vale, R.A. Milligan, The way things move: looking under the hood of molecular motor proteins, Science 288 (2000) 88-95.

[36] M. Rief, R.S. Rock, A.D. Mehta, M.S. Mooseker, R.E. Cheney, J.A. Spudich, Myosin V stepping kinetics: a molecular model for processivity, Proc. Nat. Acad. Sci. USA 97 (2000) 9482-9486.

[37] T.J. Purcell, C. Morris, J.A. Spudich, H.L. Sweeney, Role of the lever arm in the processive stepping of myosin V, Proc. Nat. Acad. Sci. USA 99 (2002) 14159-14164.

[38] M.Y. Ali, S. Uemura, K. Adachi, H. Itoh, K. Kinosita Jr., S. Ishiwata, Myosin V is a left-handed spiral motor on the right-handed actin helix, Nature Struct. Biol. 9 (2002) 464-467.

[39] Z. Ökten, L.S. Churchman, R.S. Rock, J.A. Spudich, Myosin VI walks hand-over-hand along actin, Nature Struct. Molecular Biol. 11 (2004) 884-887.

[40] D. Altman, H.L. Sweeney, J.A. Spudich, The mechanism of myosin VI translocation and its load-induced anchoring, Cell 116 (2004) 737-749.

[41] Y. Okada, N. Hirokawa, A processive single-headed motor: kinesin superfamily protein KIF1A, Science 283 (1999) $1152-1157$.

[42] K. Nishinari, Y. Okada, A. Schadschneider, D. Chowdhury, Intracellular transport of single-headed molecular motors KIF1A, Phys. Rev. Lett. 95 (2005) 118101.

[43] M. Tomishige, D.R. Klopfenstein, R.D. Vale, Conversion of Unc104/KIF1A kinesin into a processive motor after dimerization, Science 297 (2002) 2263-2267.

[44] L. Romberg, R.D. Vale, Chemomechanical cycle of kinesin differs from that of myosin, Nature 361 (1993) $168-170$.

[45] R.A. Cross, The kinetic mechanism of kinesin, TRENDS Biochem. Sci. 29 (2004) 301-309.

[46] M.L. Moyer, S.P. Gilbert, K.A. Johnson, Pathway of ATP hydrolysis by monomeric and dimeric kinesin, Biochemistry 37 (1998) 800-813.

[47] N.J. Carter, R.A. Cross, Mechanics of the kinesin step, Nature 435 (2005) 308-312.

[48] R. Lipowsky, Universal aspects of the chemo-mechanical coupling for molecular motors, Phys. Rev. Lett. 85 (2000) $4401-4404$.

[49] R. Lipowsky, N. Jaster, Molecular motor cycles: from ratchets to networks, J. Stat. Phys. 110 (2003) $1141-1167$.

[50] St.J. King, T.A. Schroer, Dynactin increases the processivity of the cytoplasmic dynein motor, Nat. Cell Biol. 2 (2000) 20-24.

[51] A.D. Mehta, R.S. Rock, M. Rief, J.A. Spudich, M.S. Mooseker, R.E. Cheney, Myosin-V is a processive actin-based motor, Nature 400 (1999) 590-593.

[52] Y. Song, E. Mandelkow, Recombinant kinesin motor domain binds to $\beta$-tubulin and decorates microtubules with a B surface lattice, Proc. Nat. Acad. Sci. USA 90 (1993) 1671-1675.

[53] S. Ray, E. Meyhöfer, R.A. Milligan, J. Howard, Kinesin follows the microtubule's protofilament axis, J. Cell Biol. 121 (1993) 1083-1093.

[54] T.M. Nieuwenhuizen, S. Klumpp, R. Lipowsky, Movements of molecular motors in two and three dimensions, Europhys. Lett. 58 (2002) 468-474.

[55] T.M. Nieuwenhuizen, S. Klumpp, R. Lipowsky, Random walks of molecular motors arising from diffusional encounters with immobilized filaments, Phys. Rev. E 69 (2004) 061911.

[56] S. Klumpp, Movements of molecular motors: diffusion and directed walks, Doctoral Thesis, University of Potsdam, 2003.

[57] T.M. Nieuwenhuizen, S. Klumpp, R. Lipowsky, Walks of molecular motors interacting with immobilized filaments, Physica A 350 (2005) $122-130$.

[58] D.C. Turner, C. Chang, K. Fang, S.L. Brandow, D.B. Murphy, Selective adhesion of functional microtubules to patterned silane surfaces, Biophys. J. 69 (1995) 2782-2789.

[59] H. Suzuki, A. Yamada, K. Oiwa, H. Nakayama, S. Mashiko, Control of actin moving trajectory by patterned poly(methylmethacrylate) tracks, Biophys. J. 72 (1997) 1997-2001.

[60] D. Riveline, A. Ott, F. Jülicher, D.A. Winkelmann, O. Cardoso, J.-J. Lacapere, S. Magnusdottir, J.-L. Viovy, L. Gorre-Talini, J. Prost, Acting on actin: the electric motility assay, Eur. Biophys. J. 27 (1998) 403-408. 
[61] Y. Hiratsuka, T. Tada, K. Oiwa, T. Kanayama, T.Q.P. Uyeda, Controlling the direction of kinesin-driven microtubule movements along microlithographic tracks, Biophys. J. 81 (2001) 1555-1561.

[62] L. Limberis, J.J. Magda, R.J. Steward, Polarized alignment and surface immobilization of microtubules for kinesin-powered nanodevices, Nano Lett. 1 (2001) 277-280.

[63] W.H. Roos, A. Roth, J. Konle, H. Presting, E. Sackmann, J.P. Spatz, Freely suspended actin cortex models on arrays of microfabricated pillars, Chem. Phys. Chem. 4 (2003) 872.

[64] R.H. Miller, R.J. Lasek, Cross-bridges mediate anterograde and retrograde vesicle transport along microtubules in squid axoplasm, J. Cell Biol. 101 (1985) 2181-2193.

[65] A. Ashkin, K. Schütze, J.M. Dziedzic, U. Euteneuer, M. Schliwa, Force generation of organelle transport measured in vivo by an infrared laser trap, Nature 348 (1990) 346-348.

[66] S.P. Gross, M.A. Welte, S.M. Block, E.F. Wieschaus, Coordination of opposite-polarity microtubule motors, J. Cell Biol. 156 (2002) 715-724.

[67] D.B. Hill, M.J. Plaza, K. Bonin, G. Holzwarth, Fast vesicle transport in PC12 neurites: velocities and forces, Eur. Biophys. J. 33 (2004) 623-632.

[68] C. Kural, H. Kim, S. Syed, G. Goshima, V.I. Gelfand, P.R. Selvin, Kinesin and dynein move a peroxisome in vivo: a tug-of-war or coordinated movement?, Science 308 (2005) 1469-1472.

[69] V. Levi, A.S. Serpinskaya, E. Gratton, V. Gelfand, Organelle transport along microtubules in xenopus melanophores: evidence for cooperation between multiple motors, Biophys. J. 90 (2006) 318-327.

[70] S.P. Gross, Hither and yon: a review of bi-directional microtubule-based transport, Phys. Biol. 1 (2004) R1-R11.

[71] M.A. Welte, Bidirectional transport along microtubules, Curr. Biol. 14 (2004) R525-R537.

[72] J.D. McGhee, P.H. von Hippel, Theoretical aspects of DNA-protein interactions: co-operative and non-co-operative binding of large ligands to a one-dimensional homogeneous lattice, J. Mol. Biol. 86 (1974) 469-489.

[73] C.T. MacDonald, J.H. Gibbs, A.C. Pipkin, Kinetics of biopolymerization on nucleic acid templates, Biopolymers 6 (1968) 1-25.

[74] A. Hoenger, M. Thormählen, R. Diaz-Avalos, M. Doerhoefer, K.N. Goldie, J. Müller, E. Mandelkow, A new look at the microtubule binding patterns of dimeric kinesins, J. Mol. Biol. 297 (2000) 1087-1103.

[75] D.T. Woodrum, S.A. Rich, T.D. Pollard, Evidence for biased bidirectional polymerization of actin filaments using heavy meromyosin prepared by an improved method, J. Cell Biol. 67 (1975) 231-237.

[76] A. Orlova, E.H. Egelman, Cooperative rigor binding of myosin to actin is a function of F-actin structure, J. Mol. Biol. 265 (1997) $469-474$.

[77] A. Vilfan, E. Frey, F. Schwabl, M. Thormählen, Y.-W. Song, E. Mandelkow, Dynamics and cooperativity of microtubule decoration by the motor protein kinesin, J. Mol. Biol. 312 (2001) 1011-1026.

[78] E. Muto, H. Sakai, K. Kaseda, Long-range cooperative binding of kinesin to a microtubule in the presence of ATP, J. Cell Biol. 168 (2005) 691-696. 